Chiva Bartoll, Ò.; Capella Peris, C. y Pallarès Piquer, M. (2018). Investigación-acción sobre un programa de aprendizaje-servicio en la didáctica de la educación física. Revista de Investigación Educativa, 36(1), 277-293.

DOI: http://dx.doi.org/10.6018/rie.36.1.270581

\title{
Investigación-acción sobre un programa de aprendizaje- servicio en la didáctica de la educación física
}

\author{
Action-research on a service-learning program \\ in teaching physical education
}

\author{
Òscar Chiva Bartoll, Carlos Capella Peris y Marc Pallarès Piquer \\ Universidad Jaume I
}

\begin{abstract}
Resumen
Introducción: El artículo relata una investigación-acción realizada sobre un programa de Aprendizaje-Servicio en el área de Didáctica de la Educación Física. El trabajo se fundamenta sobre los resultados de múltiples investigaciones previas y tiene por objetivo mejorar un programa de Aprendizaje-Servicio para favorecer la adquisición de habilidades y actitudes sociales de los futuros docentes. Para alcanzar un marco de comprensión amplio y exhaustivo sobre los efectos de la intervención planificada se empleó un método de investigación mixto. Método: por una parte, se analizó el contenido de entrevistas y diarios de seguimiento del alumnado y, por otra, se implementó un diseño cuasi-experimental con tres grupos no equivalentes. El análisis cuantitativo estuvo constituido por pruebas $t$ student para comparaciones pretest-postest, $y$ un análisis de varianza (ANOVA) para la comparación postest. Resultado: de estas pruebas se desprende que los dos grupos experimentales, al contrario que el grupo control, mejoraron significativamente sus habilidades y actitudes sociales tras sendos programas de AprendizajeServicio. Discusión: el estudio cualitativo contribuyó a complementar y mejorar la comprensión sobre los efectos del programa al matizar las habilidades y actitudes sociales logradas, que tras
\end{abstract}

Correspondencia: Óscar Chiva Bartoll, ochiva@uji.es, Avenida de Vicent Sos Baynat, s/n, 12071 Castellón de la Plana, Castellón.

Este trabajo es el resultado de una investigación procedente de un proyecto competitivo financiado (29.800 €) por el Plan de Promoción a la Investigación de la Universidad Jaume I. Código: P1-1A3013-11. 
el análisis multi-fase realizado se agrupaban en torno a tres categorías: conciencia de grupo, implicación y organización grupal, y habilidades de comunicación. Conclusiones: se concluyó que podría ser necesario mejorar el modelo de reflexión y deliberación inherente al programa, estableciéndose así las bases para una nueva planificación.

Palabras clave: investigación-acción; educación física; aprendizaje-servicio; habilidad social; universidad.

\section{Abstract}

Introduction: This article shows an action-research conducted on a service-learning program in Physical Education Didactics. The work is based on the results of multiple previous research and aims to improve the service-learning program to promote the development of social skills and attitudes on future teachers. To achieve a framework of broad and comprehensive understanding of the intervention program we used a mixed research method. Method: firstly, the content of interviews and daily monitoring diaries of students were analyzed and, secondly, it was implemented a quasi-experimental design with three nonequivalent groups. We used tests for the comparison pretest-posttest of each group, and an analysis of variance (ANOVA) for the comparison post-test. Results: the quantitative study shows that, unlike the control group, both experimental groups improved their social skills and attitudes after de service-learning program. Discussion: the qualitative study helped to complement and enhance the understanding about the effects of the program to refine the skills and attitudes achieved. The three categories obtained were: group consciousness, implication and group organization skills and communication skills. Conclusions: it was concluded that it may be necessary to improve the model of reflection and deliberation inherent in the program, thus establishing the basis for a new plan.

Key words: action research; physical education; service learning; social skills; university.

\section{Introducción}

Los cambios socioeconómicos derivados de la globalización condicionan la actual educación superior en términos de calidad, eficiencia, productividad y competitividad. Tanto es así que el Espacio Europeo de Educación Superior, indistintamente del área de conocimiento, exige una renovación pedagógica centrada en formar profesionales competentes y eficaces en el desempeño de sus funciones (Álvarez-Rojo et al., 2011; Ordóñez-Sierra \& Rodríguez-Gallego, 2015). Sin embargo, es razonable pensar que la educación universitaria no solo debe atender esta demanda, sino también educar ciudadanos comprometidos con los valores cívicos y democráticos de la sociedad en la que viven (García, Gozálvez, Vázquez \& Escámez, 2010).

En concordancia con el aprendizaje participativo, el desarrollo de competencias profesionales y la mejora de la eficiencia, el Aprendizaje-Servicio (en adelante ApS) es un método pedagógico capaz de aunar y satisfacer en gran medida estos requerimientos y, además, poner el énfasis en el desarrollo de la competencia social y ciudadana (Mayor \& Rodríguez, 2016). En el área de Educación Física el ApS es uno de los métodos pedagógicos que cosecha mayor aceptación en el ámbito universi- 
tario actual, ya que las experiencias vividas por el alumnado no solo contribuyen a la adquisición de nuevos aprendizajes, sino que ponen mayor énfasis en facilitar la formación ciudadana (Carson \& Raguse, 2014; Cervantes \& Meaney, 2013). Fundamentado en la teoría del aprendizaje experiencial de Dewey (1938), el ApS trata de establecer una relación operativa entre teoría y práctica, ofreciendo al alumnado la posibilidad de aprender mientras presta un servicio comunitario. De un modo sintético, el ApS puede definirse como una propuesta pedagógica que combina procesos de aprendizaje y servicio a la comunidad, basada en la aplicación de competencias y en la comprensión reflexiva de contenidos curriculares en contextos reales, de forma experiencial y con el objetivo de mejorar la capacidad crítica del alumnado a la vez que se ofrece un beneficio social (Eyler \& Giles, 1999; Furco \& Billig, 2002; Rodríguez-Gallego, 2014).

Una de las pruebas de su eficacia es la apuesta por los procesos de institucionalización que el ApS está protagonizando en las universidades de todo el mundo (Butin, 2006; Kenworthy-U’Ren, 2008; McIlrath, 2015). Dada esta proliferación, la necesidad de profundizar y entender mejor el ApS en el ámbito universitario es acuciante para todos los agentes comprometidos con su implantación (Aramburuzabala, Cerrillo \& Tello, 2015). En esta línea, trabajos previos como los meta-análisis de Conway, Amel y Gerwien (2009), Yorio y Ye (2012) y Warren (2012) ofrecen una aproximación panorámica del potente crecimiento del ApS en términos de investigación e implantación.

Convencidos además de que los valores y los métodos educativos no pueden ser definidos en relación con fines prefijados e invariables, ya que ello solo serviría para rebajar la educación a la categoría de racionalidad técnica (Stenhouse, 1985), esta regeneración pedagógica debe ser liderada por los agentes de la propia comunidad educativa. Para ello, es necesario un esfuerzo de indagación sistemática y de exploración autocrítica, alimentada por una actitud de búsqueda constante del profesorado (Rojas, 2009). Fiel a estos principios, el presente artículo relata una experiencia de Investigación-Acción (en adelante I-A) realizada sobre una aplicación de ApS en el área de Didáctica de la Educación Física. El objetivo principal del trabajo radica en analizar y mejorar un programa de ApS para favorecer la adquisición de habilidades $\mathrm{y}$ actitudes sociales en los futuros docentes.

Lewin (1946) definió la I-A como un proceso cíclico de exploración, actuación y valoración de resultados, con el que interpretar lo que ocurre desde el punto de vista de los actores implicados. Por tanto, la I-A se revela como uno de los modelos de investigación más adecuados para fomentar la calidad de la enseñanza e impulsar la figura del profesional investigador, reflexivo y en continua formación (Goyette \& Lesgard-Hervert, 1988; Rincón \& Rincón, 2000). Aunque el proceso de I-A ha sido descrito con diferentes matices y niveles de complejidad según diversos autores, Kemmis y McTaggart (1988) resumen el proceso en cuatro fases elementales sobre las que se fundamenta este trabajo: (1) Diagnóstico y reconocimiento de la situación inicial; (2) Desarrollo de un plan de acción, críticamente informado, para mejorar el modelo actual; (3) Actuación e investigación de los efectos en el contexto, analizados en este caso desde una aproximación investigadora mixta; (4) Discusión, reflexión y bases para una nueva planificación. 


\section{Método}

\section{Diagnóstico y reconocimiento de la situación inicial}

El diagnóstico acarrea definir el problema a resolver y declarar las intenciones de mejora, para lo que es imprescindible describir el contexto y las diferentes perspectivas existentes. En nuestro caso, el foco del problema emergía de la práctica de ApS como propuesta metodológica aplicada a una asignatura del área de Didáctica de la Expresión Corporal del Grado de Maestro/a. Con la intención de prestar un servicio directo a niños/as (con alteraciones de la motricidad) del entorno social universitario, el diseño del programa de ApS (realizado desde 2011-12), implicaba el diseño y aplicación de sesiones prácticas de juegos motores y expresivos por parte del alumnado universitario, en las que debían aplicar y desarrollar parte de los conocimientos curriculares. La parte de la asignatura impartida con ApS suponía un peso parcial de un tercio de la carga lectiva, trabajándose el resto de la asignatura con métodos de enseñanza tradicionales.

Las investigaciones que clasifican los efectos del ApS indican que estos pueden englobarse en cuatro categorías: académicos, personales, sociales y de ciudadanía (Billig, 2002; Eyler \& Giles, 1999). No obstante, como ya hemos apuntado, el objetivo de esta investigación era mejorar los efectos del programa de ApS implementado sobre el alumnado universitario a nivel social. En nuestro caso, a través de los procesos de recogida de datos propios de la I-A (Kemmis \& Mctaggart, 1988), tales como observación participante, diálogo y reuniones periódicas entre el profesorado, diarios de seguimiento, entrevistas al alumnado, grupos de discusión, cuestionarios y contacto directo con los distintos agentes implicados, hemos monitorizado minuciosamente el impacto de nuestra aplicación de ApS, obteniendo resultados favorables en las diferentes categorías de efectos establecidas.

Así pues, a nivel académico encontramos una adquisición de aprendizajes comparable al de otras metodologías de corte más tradicional (Corbatón, Moliner, Martí, Gil y Chiva, 2015; Gil, Chiva y Martí, 2015). En cuanto a los resultados de tipo personal, relacionados con cambios en los pensamientos, sentimientos, autoestima o compromiso académico, también encontramos resultados satisfactorios (Corbatón, Moliner, Martí, Gil y Chiva, 2015; Gil, Chiva y Martí, 2015). Respecto a resultados sociales, categoría que engloba todos aquellos aspectos relacionados con cambios en las habilidades para interactuar con los demás y en la toma de conciencia y comprensión de cuestiones sociales, encontramos nuevamente resultados favorables (Gil, Moliner, García, y Chiva, 2016), aunque estos estudios nos han permitido intuir que cuanto mayor ha sido la implicación del alumnado en el programa de ApS, tanto mejores han sido los resultados de la categoría de mejoras sociales. Finalmente, en la categoría de resultados de ciudadanía, que agrupa todo lo concerniente a la responsabilidad personal, la participación activa en acciones que impliquen mejoras en la comunidad, justicia, generosidad, lealtad, honestidad, etc., también hemos obtenido resultados positivos, aunque en menor medida que en las categorías anteriores (Capella, Gil, Martí y Chiva, 2015). 
Conforme al esquema de Elliot (2000), esta fase inicial de reconocimiento y diagnóstico nos lleva a identificar una situación susceptible de cambio, ya que se planteó con la intención de mejorar una práctica que ya producía resultados satisfactorios. Concretamente, el cambio que pretendíamos realizar aspiraba a mejorar las actitudes y habilidades sociales desarrolladas por el alumnado, que era el punto más débil del programa sujeto a investigación. Por tanto, aunque el ApS esté dirigido a producir un beneficio académico, comunitario y de formación en valores, en esta investigación nos centramos exclusivamente en analizar la adquisición de habilidades y actitudes sociales por parte de los futuros docentes, ya que el efecto académico y comunitario estaba contrastado y comprobado en los estudios anteriormente mencionados.

\section{Desarrollo de un plan de acción, críticamente informado, para mejorar el modelo actual}

Este paso implicó una reflexión prospectiva para diseñar las mejoras a implementar. Debía ser una propuesta viable y que en ningún caso arriesgara los beneficios conseguidos en el modelo anterior. Era necesario trazar y discutir diversas alternativas, valorando sus posibles consecuencias y, por tanto, anticipando un modelo de evaluación coherente. Para perfilar el nuevo programa se contó con la colaboración directa del alumnado de cursos anteriores, que fue interpelado sobre la cuestión. Este hecho, además de enriquecer su aprendizaje, permitió fortalecer la validez del diseño ante la potencial inestabilidad de las situaciones prácticas (Elliot, 2000).

Con el objetivo de incrementar la mejora de actitudes y habilidades sociales del alumnado, el nuevo modelo de ApS debía intensificar algunas experiencias de interacción social relacionadas tanto con la vertiente del aprendizaje como con la del servicio. Entre los cambios propuestos destacaban: la necesidad de aumentar el contacto con los receptores del servicio, incrementar la responsabilidad y la toma de decisiones, propiciar una gestión compartida del programa, ampliar el compromiso hacia el aprendizaje autónomo y en equipo, y, finalmente, arbitrar un modelo de calificación basado en la coevaluación grupal y la autoevaluación. Para asumir el importante calado de estas premisas llegamos a la conclusión de que en el nuevo modelo todo el peso de la asignatura girara en torno al programa de ApS.

Ante el sustancial giro del diseño planteado, se valoraron los riesgos y oportunidades y se decidió incorporar el nuevo modelo en modo experimental, y mantener de manera simultánea el anterior. De esta manera, ambos itinerarios coexistirían a lo largo del curso, dejando a elección del alumnado participar en uno u otro.

Por razones de organización, el número máximo de miembros asumibles por el nuevo plan de ApS se estableció en 30 alumnos. Asimismo, se optó por pautar un modelo que se iniciaría con diez sesiones teórico-prácticas en las que se informaría y formaría al alumnado sobre todos los aspectos básicos relacionados con el servicio. Primeramente, se amplió el conocimiento de los integrantes de este grupo sobre el método pedagógico del ApS, sus peculiaridades y requisitos. Seguidamente se informó al alumnado sobre las características de las diferentes entidades colaboradoras y sus niños/as, para que se distribuyeran de forma libre y equilibrada, escogiendo la entidad con la que querían colaborar. Una vez establecida la vinculación entre el alumnado y 
las entidades colaboradoras cada equipo de trabajo debía responsabilizarse de todas las actividades a realizar. Finalmente, se proporcionaron los datos de contacto de los responsables de las entidades colaboradoras para que cada grupo empezara a actuar cuando lo considerara oportuno.

Acto seguido el profesorado expuso los contenidos y competencias a trabajar en la prestación del servicio. Al mismo tiempo, también se facilitaron tres modelos de registro en los que el alumnado podría plasmar la información relativa a la aplicación del servicio, los roles desempeñados y las reuniones realizadas. Los alumnos tenían libertad para modificar o adaptar estos documentos si así lo consideraban. Por último, con la intención de estimular el proceso de reflexión durante la aplicación del servicio, también se planteó el uso de un diario de seguimiento en el que podían registrar libremente sus vivencias.

\section{Actuación e investigación de los efectos en el contexto}

Finalmente hubo un grupo de 28 universitarios que cursó la asignatura a través de lo que denominamos modelo de ApS integral-mejorado. Por su parte, otro grupo de alumnos (65 sujetos) siguió el itinerario de ApS parcial-consolidado establecido durante los cursos anteriores. La elección se vio condicionada por dos factores: (1) el orden de solicitud y (2) una pequeña argumentación motivada de la elección. En caso de que la decisión no estuviera lo suficientemente justificada el profesorado podía optar por una entrevista para dictaminar en última instancia.

Respondiendo a la naturaleza académica de la asignatura, el alumnado participante en ambos modelos de ApS organizó, aplicó y gestionó distintas sesiones de juegos motores y expresivos, a través de un servicio directo, para estimular el desarrollo motor y social de niños/as que presentaran alteraciones de la motricidad (principalmente derivadas de parálisis cerebrales leves). La diferencia fundamental entre el modelo de ApS integral-mejorado y el parcial-consolidado, tal como se concreta más adelante en la descripción de los participantes, radicaba principalmente en la interacción social con los receptores del servicio.

Posteriormente, cada grupo del nuevo modelo de ApS hizo un análisis de sus responsabilidades en la aplicación del servicio, definiendo así una hoja de ruta provisional. En este análisis se valoraron aspectos como las características de las entidades colaboradoras y sus niños/as, las diferentes estrategias de comunicación a utilizar con los responsables de los centros, los recursos disponibles en cada entidad, la detección de necesidades específicas a cubrir en cada caso, las fortalezas y debilidades de los miembros de cada equipo de trabajo, etc.

De este modo, cada equipo de trabajo procedió a delimitar sus líneas de acción en cuanto a los objetivos generales del servicio y su relación con el resto de implicados en el mismo: profesorado, profesionales de las entidades, niños/as receptores del servicio, familiares, compañeros, etc. Con el cumplimiento de estas labores se pretendía estimular el desarrollo de los aprendizajes académicos y de las habilidades y actitudes sociales del alumnado universitario.

Para indagar los efectos sobre la mejora de las actitudes y habilidades sociales del alumnado se optó por un modelo de investigación mixto, basado en la complemen- 
tariedad cuantitativa-cualitativa. Este permite triangular la información, aumentando la fiabilidad del posterior análisis, a la vez que ofrece un marco de comprensión más amplio y exhaustivo (Creswell, 2003; Ponce \& Pagán-Maldonado, 2015). Por una parte, se analizó el contenido de las entrevistas y diarios de seguimiento del alumnado y, por otra, los resultados de la aplicación de un test adaptado y validado ad hoc, a partir del cuestionario AECS-Actitudes y estrategias cognitivas sociales de Moraleda et al. (2004). Fundamentalmente, además de obtener resultados empíricos sobre estas variables, la triangulación permitiría entender mejor la naturaleza de los mismos.

A continuación, se relata la aproximación cuantitativa del estudio y, acto seguido, la cualitativa. Finalmente, en el apartado de discusión, se da cuenta de la complementariedad de los resultados, así como su contraste con otros estudios.

\section{Aproximación cuantitativa del estudio}

\section{Diseño}

Para alcanzar el objetivo de investigación de la parte cuantitativa se optó por un diseño cuasi-experimental basado en grupos naturales no equivalentes, con dos modelos de intervención constituidos según el modelo de ApS cursado y un grupo control. Se compararon los tres grupos: (1) un grupo experimental I compuesto por los integrantes del nuevo modelo de ApS "integral-mejorado"; (2) un grupo experimental II compuesto por los integrantes del modelo de ApS "parcial-consolidado"; y (3) un grupo control, compuesto por el alumnado de la asignatura que no participó en ninguno de los modelos de ApS propuestos, trabajando con métodos de corte tradicional basados en clases magistrales y prácticas entre el alumnado.

\section{Hipótesis}

Las hipótesis sobre las que se centraba la aproximación cuantitativa del estudio fueron:

a) Los dos grupos experimentales mejorarían significativamente los resultados del postest en relación con las puntuaciones del pretest.

b) El grupo experimental I (ApS integral-mejorado) obtendría mejoras significativas en el postest respecto al grupo experimental II (ApS parcial-consolidado) y al grupo control.

\section{Muestra}

La muestra del estudio estaba formada por tres grupos no equivalentes, distribuidos conforme a la elección del alumnado. Por una parte, el grupo experimental I siguió el modelo de ApS integral-mejorado, el grupo experimental II participó en el programa de ApS parcial-consolidado y el grupo control cursó la asignatura siguiendo una metodología basada en prácticas de clase tradicionales y clases teóricas magistrales (Tabla 1). 
Tabla 1

Características de los grupos analizados

\begin{tabular}{cccc}
$\begin{array}{c}\text { Grupos no } \\
\text { equivalentes }\end{array}$ & N & $\begin{array}{c}\text { Programa } \\
\text { pedagógico }\end{array}$ & Rasgos diferenciales de los grupos \\
\hline $\begin{array}{c}\text { Experimental } \\
\text { I }\end{array}$ & 28 & $\begin{array}{c}\text { ApS integral- } \\
\text { mejorado }\end{array}$ & $\begin{array}{c}\text { Mayor tiempo de contacto con los receptores } \\
\text { Responsabilidad total y participación en todas las } \\
\text { decisiones }\end{array}$ \\
Procesos de co-evaluación y auto-evaluación
\end{tabular}

\section{Instrumento}

El cuestionario empleado (Moraleda et al., 2004) mide diversas actitudes y estrategias cognitivas sociales, incluyendo un factor compuesto por variables pro-sociales que, si bien se adaptaba bastante a nuestros planteamientos, era muy extenso y requeriría de ciertas adaptaciones para ajustarlo a nuestros objetivos y a las características de los participantes. Por ello, se simplificó el cuestionario de los 137 ítems iniciales a los 41 de la versión adaptada. A continuación, indicamos las habilidades sociales de medían los ítems seleccionados y la correlación de dichos ítems en ambos cuestionarios, Tabla 2.

Tabla 2

Correlación de items y habilidades sociales en los cuestionarios

\begin{tabular}{ccc}
\hline Habilidad Social & Ítems Moraleda et al. (2004) & $\begin{array}{c}\text { Ítems cuestionario versión } \\
\text { adaptada }\end{array}$ \\
\hline $\begin{array}{c}\text { Conformidad con lo } \\
\text { socialmente correcto }\end{array}$ & $8,14,22,29,37,43,49,57$ y 65 & $1,2,3,4,5,6,7,8$ y 9 \\
\hline Sensibilidad social & $1,15,23,30,44,50,58$ y 66 & $10,11,12,13,14,15,16$ y 17 \\
\hline Ayuda y colaboración & $2,9,16,24,31,38,45,51,59$ y 67 & $\begin{array}{c}18,19,20,21,22,23, \\
24,25,26 \text { y } 27\end{array}$ \\
\hline $\begin{array}{c}\text { Seguridad y firmeza } \\
\text { en la interacción }\end{array}$ & $4,11,18,26,33,39,46,53,61$ y 69 & $\begin{array}{c}28,29,30,31,32,33, \\
34,35,36 \text { y } 37\end{array}$ \\
\hline Liderazgo prosocial & $3,10,17$ y 25 & $38,39,40$ y 41 \\
\hline
\end{tabular}


Tras esta primera selección de ítems, en arreglo a la cercanía de cada ítem con la variable que pretendía medirse, se realizó una prueba piloto para desarrollar la validación del cuestionario utilizado. En la Figura 1 presentamos el histograma de la distribución de los sujetos en esta prueba según el grado de competencia alcanzado.

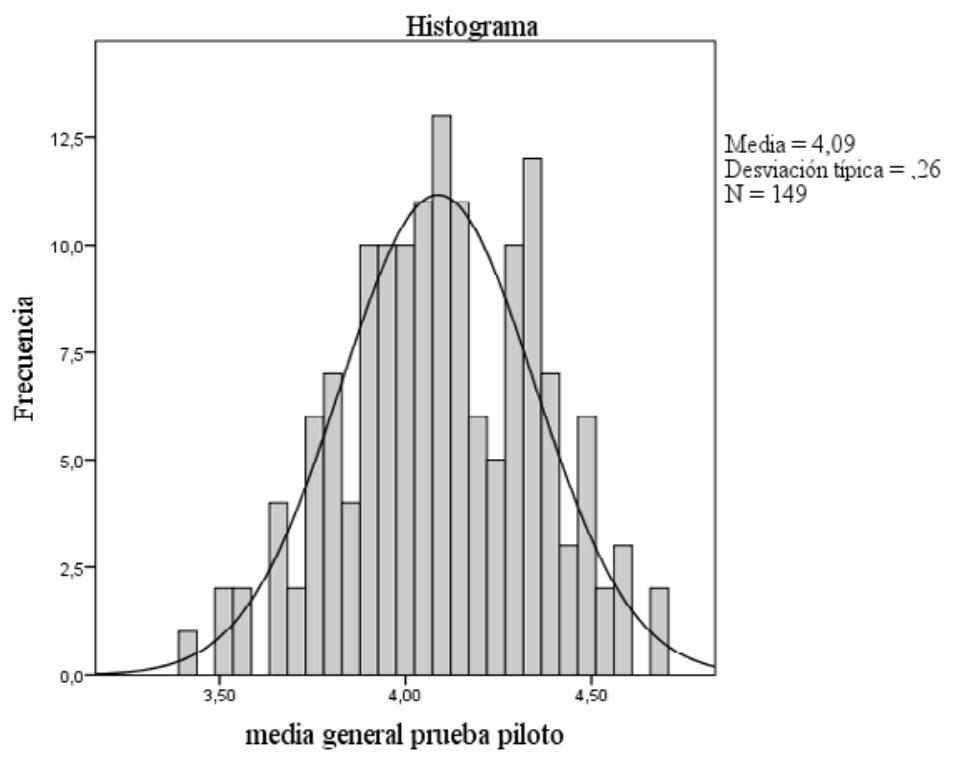

Figura 1. Histograma de distribución de los sujetos en la prueba piloto.

Por otra parte, el método de consistencia interna basado en el Alfa de Cronbach permitió estimar la fiabilidad del nuevo instrumento de medida, a través de un conjunto de ítems que se esperaba que midieran el mismo constructo o dimensión teórica (Welch y Comer, 1988). Este análisis obtuvo un valor de .859, por lo que según la categorización de George y Mallery (2003) contaba con una fiabilidad buena. Respecto al análisis factorial, el resultado para la medida de adecuación muestral de KMO (Kaiser-MeyerOlkin) resultó de 0.831 y el registro de la prueba de esfericidad de Barlett fue de .00. Por tanto, existía una correlación interna en las respuestas del alumnado. Finalmente, respecto a la estructura interna, aunque no pretendíamos analizar los distintos núcleos de contenido por separado, se aplicó el análisis de componentes principales y la normalización Oblimin con Kaiser, comprobándose que los ítems que saturaban en cada categoría coincidían con lo estipulado en el cuestionario de partida.

\section{Análisis y resultados}

En primer lugar, se comprobó la normalidad de la muestra mediante la prueba Kolmogorov-Smirnov. Posteriormente se comprobó la igualdad inicial de la muestra y se procedió a la utilización de pruebas paramétricas para contrastar las medias de los resultados. Para comprobar si existían diferencias estadísticamente significativas 
$(\mathrm{p}<.05)$ entre los análisis pre y post de cada grupo se aplicaron pruebas $T$ de student para muestras relacionadas. Por otra parte, para comparar los postest de los tres grupos se realizó un análisis de varianza (ANOVA) de un factor.

El análisis de resultados mostró que entre el pretest y el postest de los dos grupos experimentales se obtuvieron diferencias estadísticamente significativas, resultando $t(40)=2.9 ; \mathrm{p}<.05$ para el grupo experimental I y $t(40)=5.98 ; \mathrm{p}<.05$ para el grupo experimental II. Sin embargo, conforme a lo esperado, no existió una diferencia significativa entre el pretest y el postest del grupo control, con un resultado de $t(40)=1.11 ; p>.05$. De este modo confirmamos la primera hipótesis. En la Figura 2 quedan plasmados estos resultados con las puntuaciones obtenidas en cada medida, siendo evidente la mejoría en las medias del postest de los grupos experimental I y II.

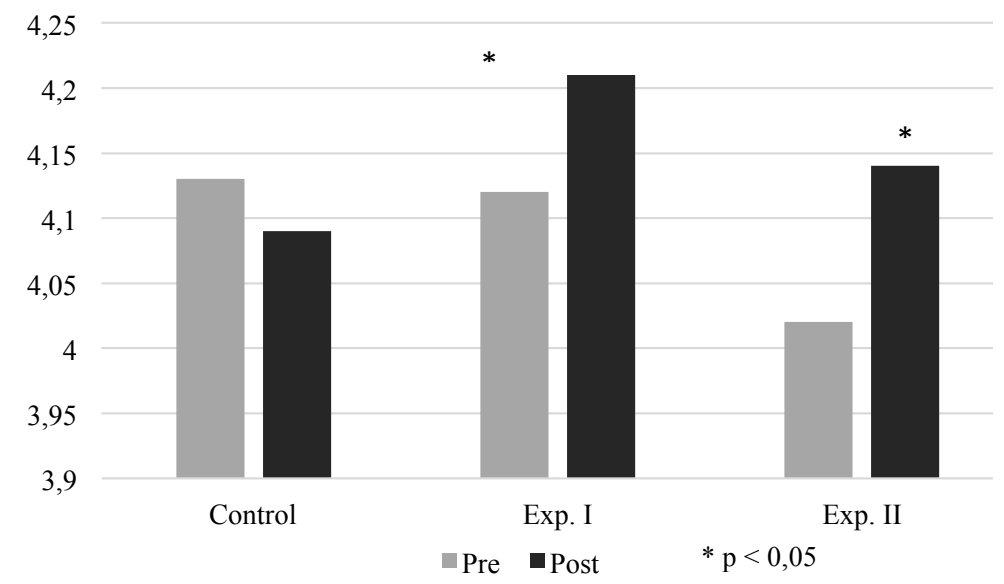

Figura 2. Evolución de habilidades y actitudes sociales de los diferentes grupos.

Por otra parte, el resultado el ANOVA reflejaría un resultado de $\mathrm{F}(2)=.38 ; \mathrm{p}>.05$, refutando así la segunda hipótesis, al no obtenerse diferencias estadísticamente significativas entre los postest de cada grupo.

\section{Aproximación cualitativa del estudio}

Los datos cualitativos se recogieron de 28 diarios de seguimiento y 15 entrevistas del grupo ApS integral-mejorado. Debido al interés por transferir los resultados a los futuros diseños de ApS se llevó a cabo un muestreo selectivo por cuota. De este modo quedaba representada la población objeto de estudio según criterios como el sexo, los rangos de edad y la experiencia previa en acciones de voluntariado. La elección de ambos instrumentos estuvo sujeta al interés de profundizar y complementar los resultados cuantitativos. Los diarios contaban con dos grandes apartados, uno referido a vivencias y experiencias personales y otro sobre aprendizajes y adquisición de competencias profesionales. Fueron recogidos al final del programa y se pidió a los participantes que antes de escribirlos reflexionaran sobre las vivencias y aprendizajes adquiridos. Por otra parte, las entrevis- 
tas fueron semiestructuradas, introduciendo preguntas abiertas en primer lugar y otras de carácter más específico posteriormente. De este modo se pretendía impedir que el marco de referencia de los investigadores se impusiera sobre los puntos de vista de los informantes. Además, la posibilidad de interpelar durante el proceso a los participantes permitió profundizar sobre algunos aspectos próximos al problema de investigación. Algunas preguntas incluidas fueron: ¿Cómo ha sido tu experiencia en el programa de ApS? ¿Cómo describirías tus interacciones con otros agentes del programa?

Todos los datos cualitativos fueron analizados a través de una aproximación multi-fase. En la fase inicial, de codificación abierta, se identificó la información relacionada con la descripción de experiencias, pensamientos y sensaciones particulares en general. Esta implicó que los investigadores analizaran las unidades de significado emergentes y que las contrastaran conforme al método de comparación constante (Patton, 2002). En la segunda fase, codificación axial, el análisis se centró en identificar de entre la información previamente codificada, aquella relacionada con las habilidades y actitudes sociales que se pretendían desarrollar. En este punto las categorías se establecían cuando empezaban a estar saturadas teóricamente (por saturación de ideas) según se identificaban patrones comunes. De esa manera, el análisis osciló entre el pensamiento inductivo y el deductivo (Flick, 2007), complementando los resultados de la parte cuantitativa del estudio. Finalmente, conforme a las indicaciones de Berglund (2005) respecto a la validez-comunicativa del análisis, existió un tercer momento de verificación intersubjetiva de las unidades de significado en el que los investigadores llevaron a cabo un proceso de member checking, sometiendo los resultados a la verificación de los alumnos informantes.

Del análisis emergieron diversos ejes de contenido. Al centrarnos en los relacionados con las actitudes y las habilidades sociales, definimos las siguientes tres categorías: (1) conciencia de grupo, (2) implicación y organización grupal y (3) habilidades de comunicación. Estas categorías, a su vez, complementan los resultados del análisis cuantitativo. De hecho, como se puede apreciar, estos tres ejes guardan una estrecha relación con los aspectos anteriormente analizados: conformidad con lo socialmente correcto, sensibilidad social, ayuda y colaboración, seguridad y firmeza en la interacción y liderazgo prosocial.

\section{Conciencia de grupo}

La experiencia de ApS ofreció al alumnado el reto de trabajar de forma cooperativa ante una exigencia real. Sus reflexiones evidencian la toma de conciencia del compromiso grupal, resaltando la importancia de realizar un esfuerzo conjunto para tener éxito en la prestación del servicio.

"Yo creo que todos los miembros del grupo tendrían que poner el mismo empeño para que todo fuera bien. [...] Entonces por mucho que lo organices y por mucho que intentes hacerlo bien, si hay una persona del grupo que no le da la gana hacerlo ya te lo fastidia todo. [...] Por eso pienso que todos los componentes del grupo se tendrían que comprometer con... igual que el resto, todas por igual, que no hubiera unas que sí y otras que no." (E.C-05-R17) 


\section{Implicación y organización grupal}

El alumnado también comprendió la necesidad de organizarse y distribuir el trabajo, no sólo a nivel cuantitativo sino también a nivel cualitativo, teniendo que decidir y repartir roles y tareas para satisfacer las necesidades de cada fase del programa.

"Lo primero fue eso, la organización entre nosotros. [...] Lo primero que se nos ocurrió fue hacer roles, igual que hacemos los roles para las sesiones escritas hicimos roles en los juegos. Cada uno tenía un rol que cumplir en cada momento." (E.C-03-R4)

Esta capacidad de organización también hizo acto de presencia a nivel intrapersonal al exigir al alumnado la competencia de resolver cuestiones para controlar todo tipo de contingencias derivadas de la interacción con otros.

"He tenido que planificar sesiones, he tenido que estar allí y explicarlas, improvisar juegos que no... que habíamos pensado una cosa y luego hemos llegado allí y o no salían o se nos quedaba corta la sesión teníamos que hacer juegos otros o que no salían, no los entendían... entonces tenías que o modificarlos para hacerlos más sencillos o elegir otro tema, otro juego y modificarlo..." (E.C-08-R2)

\section{Habilidades de comunicación}

La interacción con los diferentes agentes implicados en el programa de ApS obligó al alumnado a comunicarse y entenderse. Por una parte, sus discursos aluden al desarrollo que la experiencia les proporcionó para comunicarse y establecer relaciones empáticas con los niños/as.

"Pero luego ya... [...] a mí por ejemplo no me resulta ya difícil llegar y hablar con ellos. Por ejemplo, de la clase siempre los saco yo, y hacemos el tren y [...] cantamos la cancioncita y yo no tengo ningún problema en estar con ellos." (E.C-08-R9)

Por otra, el alumnado hizo referencia explícita a su competencia para establecer líneas de comunicación y contacto afectivo, cada vez más fluidas, con otros agentes del programa. Un ejemplo paradigmático es la relación con los padres.

"Yo al principio, en la primera sesión no, pero luego ya sí que fui a hablar con varios padres. Y luego la última sí que hable con todos e incluso nos hicimos fotos y todo, hubo, hubo bastante contacto. Que sí que al principio te cuesta, porque no sé, son sus hijos y a lo mejor vienen y te sientes un poco coaccionado." (E.C-012-R7)

Finalmente se destacó la mejora de su capacidad de argumentación y respeto para con las opiniones de los demás. Aprendiendo, además, a poner todo este aprendizaje a disposición de los objetivos grupales. 
"Al principio no era muy consciente de todo esto, pero después de reflexionarlo y de pensarlo y eso pues me he dado cuenta de que realmente sí que he aprendido muchas cosas a través de esta experiencia. Y bueno, por una parte trabajando en grupo pues me he dado cuenta de que he mejorado mi capacidad para argumentar las cosas que hago, pues que he adquirido también actitudes de respeto hacia las diferentes opiniones de mis compañeros, también pues compromiso grupal, responsabilidad, aquello que te decía al principio también me he dado cuenta de que tiene mayor rendimiento trabajar en grupo que no de forma individual y competitiva, y bueno también de la importancia de los roles para que salga bien el trabajo." (E.C-09-R6)

\section{Discusión, reflexión y bases para una nueva planificación}

De la complementariedad proporcionada por el enfoque investigador mixto se desprenden argumentos para determinar que las habilidades y actitudes sociales del alumnado mejoraron gracias a la vivencia de ambos modelos de ApS. Estos argumentos se sustentan tanto sobre la comparación pretest - postest del análisis cuantitativo, como en el análisis cualitativo realizado sobre el discurso de los implicados. No obstante, en la comparación entre los postests del cuestionario las diferencias no fueron significativas entre el grupo experimental I y el II, refutándose así la segunda hipótesis del enfoque cuantitativo del estudio. Por este motivo, con los resultados obtenidos, es difícil reconocer y admitir que el ajuste del nuevo modelo de ApS fue plenamente satisfactorio, por lo que debemos seguir trabajando para seguir mejorando el modelo propuesto. Sin embargo, aunque del análisis cuantitativo no puede inferirse estadísticamente que el ApS integral-mejorado implicara un mejor escenario para el desarrollo de habilidades sociales, en la aproximación cualitativa se recogen referencias constantes sobre la mejora de habilidades y actitudes sociales en los participantes del nuevo modelo de ApS, reflejando al menos una tendencia clara de mejora.

En términos generales, nuestra investigación coincide con numerosos estudios sobre los efectos del ApS que reportan mejoras en las habilidades sociales del alumnado (Conway et al., 2009; Eyler \& Giles, 1999; Warren, 2012; Yorio \& Ye, 2012). Efectos que se concretan además en el ámbito de la educación física y su didáctica, tal como muestran las revisiones de Carson y Raguse (2014) y Cervantes y Meaney (2013). Aunque es difícil asegurar que la mejora producida provenga del cambio del contexto de interacción, sí entrevemos una clara tendencia en este sentido. De un modo similar Weiler, La Goy, Crane y Rovner (1998) evidencian mejoras en actitudes de interacción social; Astin y Sax (1998) también coinciden con este planteamiento a raíz de estudios cuantitativos y cualitativos efectuados de forma longitudinal sobre alumnado de diferentes niveles educativos; Billig (2002) y Conrad y Hedin (1989) lo reafirman a partir de sendas revisiones bibliográficas basadas en los efectos del ApS en diferentes contextos y niveles educativos; y, finalmente, Eyler y Giles (1999) apuntan conclusiones similares en estudios basados en encuestas y entrevistas en profundidad a estudiantes de diferentes instituciones.

Manteniendo una relación todavía más próxima con las características de nuestro estudio, Maynes, Hatt y Wideman (2013) apuntan mejoras en la actitud social del alumnado, apoyándose en una investigación realizada con alumnos del Grado de Maestro/a. 
Estos autores destacan que, además del escenario de interacción social, la importancia del proceso de reflexión podría constituir un elemento crucial para la mejora de habilidades y actitudes sociales. Este enfoque, apoyado sobre las bases constructivistas del aprendizaje experiencial, aporta una potencial explicación a nuestros resultados. En particular podría haberse dado el caso de que, al carecer nuestro programa integral-mejorado de ApS de procesos rigurosos y sistemáticos de deliberación y reflexión, el alumnado no hubiera llegado a interiorizar y tomar conciencia plena de este tipo de aprendizajes. Este hecho explicaría por qué en los instrumentos cualitativos de recogida de datos sí existió alusión explícita y reiterada a dichos aprendizajes, mientras que desde el enfoque cuantitativo no se alcanzaron diferencias significativas en todas las comparaciones postest. El quid de la cuestión podría encontrarse en la mayor reflexión que los diarios y las entrevistas, a diferencia de los cuestionarios, exigen a la hora de contestar. Por ello, los resultados obtenidos desde la aproximación cualitativa desvelan cuestiones que los cuestionarios podrían haber pasado por alto. Este hecho pone de manifiesto el valor complementario del método mixto, ya que permite interpretar más profundamente los resultados.

En cualquier caso, este descubrimiento reclama la necesidad de incorporar nuevas dinámicas de reflexión y deliberación para el alumnado, con el objetivo de favorecer, además de su capacidad deliberativa, la interiorización de los aprendizajes. En este sentido, Englund (2015) establece que un proceso deliberativo en educación implica cuestiones que, a nuestro juicio, deberían cumplirse necesariamente en el nuevo modelo de ApS. Entre ellas destacamos:

- Confrontación de diferentes puntos de vista ante la toma de cada decisión

- Marco de tolerancia y respeto constante

- Aspiración inicial de alcanzar consensos

- Cuestionamiento de la autoridad y/o la tradición

En cuanto a los principios a seguir para fomentar procesos de reflexión en la formación de futuros docentes, coincidimos con Labarrere (1996) postulando los siguientes:

- Estilo comunicativo del profesorado

- Papel de las vivencias en la formación

- Papel de los métodos pedagógicos

- Reflexión como procedimiento de los propios métodos pedagógicos

En alusión a este último, nuestra misión conforme al esquema de acción seguido (Kemmis y McTaggart, 1988) consistiría por tanto en diseñar un nuevo modelo de ApS que integrara procedimientos reflexivos con los que el alumnado pudiera interiorizar el aprendizaje, a través de un proceso interactivo de deliberación y reflexión conjunta.

\section{Conclusiones y consideraciones finales}

En primer lugar, queremos destacar que el proceso de I-A desarrollado, sustentado en los resultados de múltiples investigaciones previas, ha proporcionado información de gran relevancia para mejorar el programa de ApS diseñado y aplicado en una 
asignatura del área de Didáctica de la Educación Física. La obtención de mejoras significativas en los dos grupos experimentales destaca las contribuciones positivas de los modelos de ApS aplicados (integral-mejorado y parcial-consolidado) en la mejora de habilidades y actitudes sociales. Por otra parte, aunque existe una tendencia de mejora respaldada por las mejores puntuaciones medias del alumnado que cursó el programa integral-mejorado, la ausencia de diferencias significativas en la comparación postest-postest de los dos grupos experimentales aconseja seguir mejorando el diseño. Además de reforzar estas valoraciones, los resultados del estudio cualitativo aportan una visión de gran importancia para mejorar nuestra comprensión sobre el proceso vivenciado por los alumnos al participar en la experiencia de ApS propuesta. En conclusión, se detecta la necesidad de mejorar el modelo actual planteando nuevos escenarios que potencien la reflexión y deliberación sobre las decisiones a tomar, tanto a nivel individual como grupal.

Tras este proceso de I-A se hace patente que orientar la praxis docente es una responsabilidad que atañe tanto a los docentes como a los investigadores especializados. La investigación educativa no puede conformarse con el uso generalizado de pruebas cuyas respuestas aspiren siempre a extrapolarse con afán universalizador. Si se aceptaran estos criterios, la buena praxis docente pasaría a ser una mera cuestión de eficacia técnica que, en el mejor de los casos, se basaría en la aplicación diligente de reglas contingentes y descontextualizadas (Elliot, 2000). Desde esta perspectiva, se concluye que la docencia y la investigación educativa no son actividades distintas.

\section{Referencias}

Álvarez-Rojo, V., Romero, S., Gil-Flores, J., Rodríguez-Santero, J., Clares, J., Asensio, I., . . . Salmeron-Vilchez, P. (2011). Necesidades de formación del profesorado universitario para la adaptación de su docencia al espacio europeo de educación superior (EEES). RELIEVE, 17(1), 1-22.

Aramburuzabala, P., Cerrillo, R., \& Tello, I. (2015). Aprendizaje-servicio: una propuesta metodológica para la introducción de la sostenibilidad curricular en la universidad, Profesorado, 19(1), 78-95.

Astin, A. W., \& Sax, L. J. (1998). How undergraduates are affected by service participation. Journal of College Student Development, 39(3), 251-263.

Berglund, H. (2005). Toward a Theory of Entrepreneurial Action, (Tesis Doctoral, Chalmers University of Technology). Recuperado de http://www.henrikberglund.com/thesis/ index.htm

Billig, S. H. (2002). Support for K-12 service learning practice: A brief review of the research. Educational Horizons, 80(4), 184-189.

Butin, D. W. (2006). The limits of Service-Learning in Higher Education. The Review of Higher Education, 29(4) 473-498.

Capella, C.; Gil, J.; Martí, M. y Chiva, Ó. (2015). Estudio de caso múltiple con historias de vida en el grado de educación infantil: aprendizaje-Servicio en la didáctica de la educación física. Profesorado, 1(19), 334-348.

Carson, R. L., \& Raguse, A. L. (2014). Systematic Review of Service-Learning in Youth Physical Activity Settings. Quest, 66(1), 57-95. 
Cervantes, C. M., \& Meaney, K. S. (2013). Examining service-learning literature in physical education teacher education: Recommendations for practice and research. Quest, 65(3), 332-353.

Conrad, D., \& Hedin, D. (1989). High school community service: A review of research and programs. Madison, WI: National Center on Effective Secondary Schools.

Conway, J. M., Amel, E. L., \& Gerwien, D. P. (2009). Teaching and Learning in the Social Context: A Meta-Analysis of Service Learning's Effects on Academic, Personal, Social, and Citizenship Outcomes. Teaching of Psicology, 36(4), 233-245.

Corbatón, R.; Moliner, L.; Martí, M.; Gi, J. y Chiva, Ó. (2015). Efectos académicos, culturales, participativos y de identidad del Aprendizaje-Servicio en futuros maestros a través de la educación física. Profesorado, 1(19), 280-297.

Creswell, J. W. (2003). Research Design. Qualitative, quantitative and mixed methods approaches. London: Sage.

Dewey, J. (1938). Experience and education. New York, NY: Macmillan.

Elliot, J. (2000). Cambio educativo desde la investigación-acción. Madrid: Morata.

Englund, T. (2015). Towards a deliberative curriculum? Nordic Journal of Studies in Educational Policy, 1(1), 48-56.

Eyler, J., \& Giles, D. E. (1999). Where's the learning in service-learning? San Francisco: Jossey-Bass.

Flick, U. (2007). Introducción a la investigación cualitativa. Madrid: Morata.

Furco, A., \& Billig, S. H. (2002). Service Learning. The essence of the Pedagogy. Greenwich, CT: Information Age Publishing.

García, R., Gozálvez, V., Vázquez, V., \& Escámez, J. (2010). Repensando la Educación: cuestiones y debates para el siglo XXI. Valencia: Brief Ediciones.

George, D., \& Mallery, P. (2003). Spss for Windows step by step: A Simple Guide and Reference. 11.0 Update. Boston: Allyn \& Bacon.

Gil, J., Chiva, Ó., y Martí, M. (2015). The impact of service learning on the training of pre-service teachers: Analysis from a physical education subject. European Physical Education Review, 21(4), 467-484.

Gil, J., Moliner, O., García, R., y Chiva, O. (2016). Una experiencia de AprendizajeServicio en futuros docentes: desarrollo de la competencia social y ciudadana. Revista Complutense de Educación, 27(1), 53-73.

Goyette, G., \& Lessard-Hérbert, M. (1988). La investigación-acción. Funciones, fundamentos e instrumentación. Barcelona: Alerte.

Kemmis, S., \& McTaggart, R. (1988). Cómo planificar la investigación-acción. Barcelona: Laertes.

Kenworthy-U'Ren, A. L. (2008). A review of the field of ten years after JOBE's seminal special issue. Journal of Business Ethics, 81, 811-822.

Labarrere, A. (1996). Pensamiento. Análisis y autorregulación de la actividad cognoscitiva de los alumnos. C. Habana: Pueblo y Educación.

Lewin, K. (1946). Action research and minority problems. Journal of Social Issues, 2, 34-46.

McIlrath, L. (2015). La Universidad Cívica ¿Un vacío legal y político? Profesorado, 19(1), 26-40.

Maynes, N., Hatt, B., \& Wideman, R. (2013). Service Learning as a Practicum Experience in a Pre-Service Education Program. Canadian Journal of Higher Education, 43, 80-99. 
Mayor, D., \& Rodríguez, D. (2016). Aprendizaje-Servicio y práctica docente: una relación para el cambio educativo. Revista de Investigación Educativa, 34(2), 535-552. doi: http://dx.doi.org/10.6018/rie.34.2.231401

Moraleda, M., González, A., \& García-Gallo, J. (2004). AECS: actitudes y estrategias cognitivas sociales. Madrid: TEA.

Ordóñez-Sierra, R., \& Rodríguez-Gallego, M. R. (2015). Docencia en la universidad. Valoraciones de los estudiantes de la Universidad de Sevilla. Bordón. Revista de Pedagogía, 67(3), 85-102. doi:10.13042/Bordon.2015.67305

Patton, M. Q. (2002). Qualitative research and evaluation methods (3rd Ed.). Thousand Oaks, CA: Sage.

Ponce, O., \& Pagán-Maldonado, N. (2015). Mixed Methods Research in Education: Capturing the Complexity of the Profession. International Journal of Educational Excellence, 1(1), 111-135.

Rincón, D., \& Rincón, B. (2000). Revisión, planificación y aplicación de mejoras. Revista Interuniversitaria del Profesorado, 39, 51-73.

Rodríguez-Gallego, M. R. (2014). El Aprendizaje-Servicio como estrategia metodológica en la Universidad. Revista Complutense de Educación, 25, 95-113. doi: 10.5209/ rev_RCED.2014.v25.n1.41157

Rojas, A. E. (2009). La investigación-acción en el aula. Comunicación presentada en la Conferencia Iberoamericana de Ministros de Educación: Metas educativas 2021. Recuperado de http://www.oei.es/metas2021/reflexiones2/10.pdf

Stenhouse, L. (1985). Investigación y desarrollo del curriculum. Morata: Madrid.

Warren, J. L. (2012). Does Service-Learning Increase Student Learning? A Meta-Analysis. Michigan Journal of Community Service Learning, 18(2), 56-61.

Weiler, D., La Goy, A., Crane, E., \& Rovner, A. (1998). An evaluation of K-12 service-learning in California: Phase II final report. Emeryville, CA: RPP International.

Welch, S. \& Comer, J. (1998). Quantitative methods for public administration: techniques and applications. Virginia: Brooks/Cole Pub. Co.

Yorio, P., \& Ye, F. (2012). A Meta-Analysis on the Effects of Service-Learning on the Social, Personal, and Cognitive Outcomes of Learning. Academy of Management Learning \& Education, 11(1), 9-27.

Fecha de recepción: 10 de octubre de 2016.

Fecha de revisión: 9 de diciembre de 2016.

Fecha de aceptación: 9 de diciembre de 2016. 
